\title{
Roles of Vitamin Metabolizing Genes in Multidrug-Resistant Plasmids of Superbugs
}

Asit Kumar Chakraborty, Genetic Engineering Laboratory, Department of Biotechnology \& Biochemistry, Oriental Institute of Science \& Technology, Vidyasagar University, Midnapore 721102, West Bengal, India.

\begin{abstract}
Superbug crisis has rocked this world with million deaths due to failure of potent antibiotics. Thousands mdr genes with hundreds of mutant isomers are generated. Small integrons and R-plasmids have combined with $F^{\prime}$-plasmids creating a space for $>$ 10-20 of mdr genes that inactivate antibiotics in different mechanisms. Mdr genes are created to save bacteria from antibiotics because gut microbiota synthesize $>20$ vitamins and complex bio-molecules needed for $>30000$ biochemical reactions of human metabolosome. In other words, mdr gene creation is protected from both sides, intestinal luminal cells and gut bacteria in a tight symbiotic signalling system. We have proposed, to avert the crisis all vitamin metabolizing genes will be acquired in MDRplasmids if we continue oral antibiotics therapy. Therefore, we have checked the plasmid databases and have detected thiamine, riboflavin, folate, cobalamine and biotin metabolizing enzymes in MDR plasmids. Thus vit genes may mobilise recently into MDR-plasmids and are likely essential for gut microbiota protection. Analysis found that $c o b$ and thi genes are abundant and likely very essential than other vit genes.
\end{abstract}

Keywords: vitamin synthesizing genes, MDR plasmids, gut microbiota, superbugs

\section{Introduction}

\section{MDR bacteria contaminated in air dust and rain water}

Life creation is due to chemical evolution and Darwin's adaptation theory tells the unique way of changes in cellular structure and metabolism in different eco-system. Microscopic unicellular bacteria, fungi, yeast, protozoa, and algae are regulating water and atmosphere circling plant and animal kingdoms (Pathak et al., 1993; Ahmadjian, 2000). A battle among the creatures is maximum at all points where simple bacteria create crisis in the human life creating toxins and other metabolic effects (Grossart et al., 2013; Mckenna, 2013). Microbes are in symbiotic relation with human and $2 \times 10^{12}$ gut bacteria perform various syntheses of bio-molecules like vitamins, butyrate, unsaturated fatty acids, bile salt and prostaglandin (Le Chatelier et al., 2013). Recently, multidrug-resistant microbes have increased in global water and atmosphere (PM10 particulates increased) posing a threat to human population 
worldwide (Ibiene et al., 2011; Chakraborty, 2017b). Antibiotics had cured most infections between1940-1990 although gradual increase in drug resistance was detected in many continents as early as 1960 (Reynolds , 1989; Paul et al., 2013). Indeed the drug industry had always run to discover potent derivatives like cefotaxime, ceftrioxane, imipenem and dorripenem in place of old drug like ampicillin and oxacillin (Laxminarayan et al., 2013). Most harmful changes have happened when 2-15 kb R-plasmids and integrons are combined with $62 \mathrm{~kb} \mathrm{~F}$-plasmids and such MDR conjugative plasmids donate mdr genes into all bacteria more easily by conjugation (Chakraborty, 2016a). Thus, $40 \%$ common bacteria in Ganga river water and Bay of Bengal sea water are ampicillin resistant and most bacteria (>95\%) have isolated from human and animal are ampicillin and tetracycline resistant being two early mdr genes (amp and tet that have sequenced in 1965 as plasmid pBR322) and many mdr genes are detected in plasmids of intestinal bacteria (Morten et al., 2009).

\section{Beta-lactamase genes are strongly diversified}

Most notorious MDR gene is $\beta$-lactamase gene (accession nos. J01749, X92506, AF227505, AF124204) which hydrolyses lactam ring CO-N bond of penicillins and has diversified into 20 different classes (250-382aa) like blaTEM, blaOXA, blaCTX-M, blaCMY, blaKPC and blaNDM-1etc (Bush \& Jacoby, 2010; Chakraborty, 2016b; Stiffler et al., 2015). Class B enzymes are $\mathrm{Zn++-dependent} \beta$-lactamases that demonstrate a hydrolytic mechanism different from that of the serine $\beta$-lactamases of classes A, C, and D. Organisms producing these enzymes usually exhibit resistance to penicillins, cephalosporins, carbapenems, and the clinically available $\beta$-lactamase inhibitors. Class C AmpC $\beta$-lactamases include CMY-2, P99, ACT-1, and DHA-1, which are usually encoded by bla genes located on the bacterial chromosome, although plasmid-borne AmpC enzymes are becoming more prevalent. Moreover, OXA-2, OXA-23, OXA-48 and OXA-51/58 have no sequence similarity but all hydrolyses carbapenems and some inhibitors. The Klebsiella pneumoniae New Delhi metallo- $\beta$-lactamase-1 (NDM-1) was discovered in 2009 in an Indian patient that could hydrolyse all $\beta$-lactums (Mataseje et al., 2016). However, NDM1 outbreaks in England and USA as early as 2010 and were found in plasmid as well as chromosome of $K$. pneumoniae, E. coli and Acinetobacter baumannii as well as to lesser extent in Providentia sp. and Enterobacter sp.(Chakraborty, 2016a).

\section{Other major mdr genes that inactivate antibiotics}


StrA/B gene (accession nos. KT225462, LN555650) encodes an enzyme ( 267aa) that acetylates streptomycin and diversified aac (accession nos. AB061794, JN596279) and aph (accession nos. X01702, U32991) mdr genes acetylate and phosphorylate aminoglycoside antibiotics respectively which then could not able to bind ribosome to kill bacteria (Shaw et al., 1993; Villa et al., 2015). The aminoglycoside adenyl transferase [EC:2.7.7.47] was present in many bacterial plasmids like Escherichia (accession nos. HG41719, KJ484637, KM377239), Klebsiella (accession nos. KF914286, KF719970), Salmonella (accession no. JQ899055), Acinetobacter (accession no. KM401411), but also present in some bacterial chromosome as in Salmonella enterica. Cat gene (accession no. EF516991) acetylates chloramphenicol and acetylated chloramphenicol could not bind 30S ribosome (Robicsek et al., 2006; Schwarz et al., 2004). Sul1/2 genes (accession nos. KM877269, AP012056) have been implicated in sulfamethoxazole resistance (Dallas et al., 1992) and arr3 gene (accession no. KX029331; protein id. APD70456) ribosylates refamycins which then could not inhibit bacterial RNA polymerase (Chakraborty, 2016a).

\section{Drug efflux genes are accumulating in large plasmids}

Major drug efflux MDR genes include tet gene isomers which encode a membrane-bound drug efflux protein ( 400aa) which kicks out tetracycline from bacterial cell cytoplasm and also has been diversified into tetA/B/C (accession nos. X75761, KC590080) (McMurry et al., 1980). Other potential genes are MFS, RND and MATE types drug efflux genes that could kick out drugs in a proton-pump mechanism (Sun et al., 2014). The MexAB-OprM system in Pseudomonas aeruginosa has the broadest substrate specificity and contributes to resistance to macrolides, aminoglycosides, sulfonamides, fluoroquinolones, tetracyclines and many $\beta$ lactams. The loss of the outer membrane protein (porin) OprD, is associated with imipenem resistance and reduced susceptibility to meropenem. Similarly, tetracycline resistant protein, tetM (accession no. AY466395, protein id. AAS45561) binds tetracycline increasing drug MIC (Croft et al., 2013; Wang et al., 2015; Chakraborty, 2016a).

\section{Target mutations are prominent mechanism for multi-resistance}

The majority of rifampicin-resistant clinical isolates of $M$. tuberculosis harbour mutations in the 507-533 coding region of $r p o B$ gene creating an altered $\beta$-subunit of the RNA polymerase that could not able to bind refampicin and altered KatG gene (S315T mutation) and -15C/T mutation in the promoter may be important in isoniazid resistance targeting NADHdependent enoyl-acyl carrier protein (ACP)-reductase that is involved in mycolic acid 
synthesis. VanA gene cluster are involved in the vancomycin resistance in Enterococcus facium (Merlo et al., 2015; also see, plasmids pIP501 and pAM_1) and ermA/B genes are diverged 23S rRNA methyl transferases that give resistant to macrolides (Harme et al., 2015). This implies that bacteria do change its target genes if new mdr gene synthesis is delayed or very high dose antibiotic is used. So bacteria need multiple gateways to inactivate drug quickly to save gut bacteria (Chakraborty, 2016c).

\section{Variety of large plasmids in multidrug-resistant bacteria}

BlaKPC gene was located in many Klebsiella pneumoniae large (108-317kb) conjugative plasmids (accession nos. NC_022078, NC_014312 and JX283456) as well as NDM1 gene was also located in conjugative plasmids (accession nos JN420336, AP012055). Other Escherichia coli plasmid pNDM_Dok01 and $K$. pneumoniae plasmid pKpANDM-1 have flanked ISAba125 elements and T4SS genes (Hu et al., 2012; Feng et al., 2015; Flach et al., 2015). Folster JP et al (2014) reported a Vibrio cholera strain 2012EL-2176 harbouring IncA/C2 plasmid containing blaCMY-2, blaCTX-M-2, blaTEM-1, floR, aac(3)-IIa , strA/B, sul1/2, dfrA1, dfrA27, tetA, mphA, mdr-genes and also resistant to ciprofloxacin due to mutation in $\operatorname{gyr}(\mathrm{S} 83 \mathrm{I}) / \operatorname{parC}(\mathrm{S} 85 \mathrm{~L})$ as also seen in plasmid pMRV150 (accession no. EU116442) (Carattoli, 2009; Huang et al., 2013; Hermi et al., 2015).

\section{MDR Genes move into chromosome to increase gene dose}

In skin infection, methicillin resistance gene ( $m e c A)$ encoding a penicillin-binding protein and activated with mobile genetic element, the staphylococcal cassette chromosome mec (SCCmec) has been reported in Staphylococcus aureus chromosome which also is associated with bla, aac, aad and sul1/2 types MDR genes. This implies that to avert the drug crisis, $m d r$ genes have been mobilized into chromosome to increase in copy number and in association with multiple $m d r$ genes (Chakraborty, 2016b).

\section{Vitamin metabolizing genes in bacteria}

Escherichia coli K-12 synthesizes thiamine monophosphate (vitamin B1) de novo from two precursors, 4-methyl-5-(beta-hydroxyethyl) thiazole monophosphate and 4-amino-5hydroxymethyl-2-methylpyrimidine pyrophosphate. Thiamine monophosphate is then phosphorylated to make thiamine pyrophosphate coenzyme. Five tightly linked genes 
(thiCEFGH) are involved and the thiC gene product is required for the synthesis of the hydroxymethylpyrimidine and thiE, thiF, thiG, and thiH gene products are required for synthesis of the thiazole. Rizobium plasmids carry many thi genes with $\sim 38-69 \%$ similarities to E. coli enzymes (Vander Horn et al., 1993; Rodionov et al., 2002). RibB gene (protein ids. CDQ55093; ABU50713) of Klebsiella pneumoniae has similarity to 3,4-dihydroxy-2butanone 4-phosphate synthase and linked to ribA (protein id. ABU50715), ribH (protein id. ABU50714), ribE (protein id. ABU50712) of Photobacterium kishitanii (accession no. AY849504.2) and ribX is riboflavin transport system permease (protein ids. GAX64964, AUS72405) and ribY riboflavin binding protein (protein id. GBD30789) (Lopez et al., 1987; Dallas et al., 1992; Talarico et al., 1991). The complete aerobic and anaerobic pathways for the de novo biosynthesis of B12 are known including lower ligand or 5,6dimethylbenzimidazole (Hazra et al., 2015).

We have extensively reviewed the beta-lactamases (Chakraborty, 2016b) and drug acetyl transferases (Chakraborty et al., 2017) in plasmids and integrons and their association in other mdr genes (Chakraborty, 2016a; Chakraborty, 2017c,f). From GenBank data analysis, we have observed an increase in drug efflux genes and IS-elements in MDR conjugative plasmids with reducing the TRA conjugative genes. Which means most of the bacteria have now many types of MDR-plasmids and as we are continuing insult with complex oral antibiotics, other necessary changes are obvious (1/4 genes in MDR-plasmids are unknown function) to avert the serious health crisis of human and bacteria under strong symbiosis (Ame J Drug Deli Ther., 2018, in press). So we have investigated here the nature of vitamin synthesizing genes in large MDR conjugative plasmids to prove that superbug horror and antibiotic horror are synonemous and many changes have occurred in bacterial genomes and plasmids as we have increased multiple complex antibiotics doses since 1960s.

\section{Materials and Methods}

Water from Ganga River was collected at the morning from Babu Ghat (Kolkata-700001) and Howrah Station area. About $100 \mu$ l of water was spread onto 1.5\% Luria Bartoni-agar plate containing different concentration of antibiotics at $2-50 \mu \mathrm{g} / \mathrm{ml}$. MDR bacteria were selected in agar-plate containing ampicillin, streptomycin, chloramphenicol, tetracycline or ciprofloxacin at $50,50,34,20 \mu \mathrm{g} / \mathrm{ml}$ respectively. As imipenem and meropenem resistant bacteria were 
present low (0.08-0.2 cfu/ml water), a modified method was followed. $2 \mathrm{ml} 5 \mathrm{x} \mathrm{LB}$ media was added into $10 \mathrm{ml}$ River/Sea water at $2-10 \mu \mathrm{g} / \mathrm{ml}$ concentration and was incubated $24 \mathrm{hrs}$ to get drug resistant bacteria population (Chakraborty, 2015). Meropenem resistant bacteria further selected on ampicillin, tetracycline, chloramphenicol and streptomycin to get the superbugs. Antibiotics were purchased from HiMedia and stored at $2-50 \mathrm{mg} / \mathrm{ml}$ at $-20^{\circ} \mathrm{C}$. Antibiotic papers were also purchased from HiMedia according to CLSI standard. Antibiotic papers are:

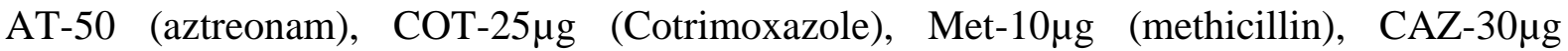

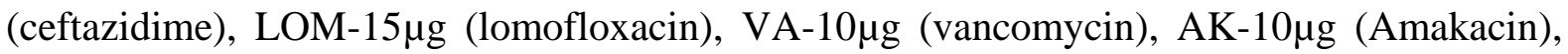

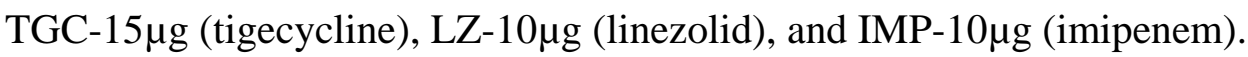

The plasmid DNA was isolated from overnight culture using Alkaline-Lysis Method (Sambrook et al., 1982; Chakraborty \& Hodgson, 1988). 16S rDNA gene colour Sanger's dideoxy sequencing was performed by SciGenom Limited, Kerala, India (Ausubel et al., 1989). PCR amplification was performed using 1 unit Taq DNA polymerase, 20ng DNA template, $0.25 \mathrm{mM}$ dXTPs, $1.5 \mathrm{mM} \mathrm{MgCl}_{2}$, for 35 cycles at $95^{\circ} \mathrm{C} / 30$ " (denaturation)$52^{\circ} \mathrm{C} / 50$ "'(annealing)- $72^{\circ} \mathrm{C} / 1.5^{\prime}$ (synthesis). The product was resolved on a $1 \%$ agarose gel in $1 \mathrm{X}$ TAE buffer at 50V for 2-4 hrs and visualized under UV light and photograph was taken (Chakraborty et al., 1993). The 16S rRNA gene amplification and mdr genes sequencing are performed by conventional methods (Sanger et al., 1977; Chakraborty et al., 1991). NCBI BLAST analysis was performed for bacterial specific gene analysis (www.ncbi.nlm.nih.gov/blast) and data was submitted to GenBank. NCBI databases were retrieved using the BLAST programmes (www.ncbi.nlm.nih.gov/blast) (Johnson et al., 2008).

The complete genes are sequenced in plasmids and were analyzed by Seq-2 programme of BLAST. Multalin protein sequence software was used to get the nature of conserved sequences among metallo-class B $\beta$-lactamases (King and Strynadka, 2013). Sometime, diverged sequences are manually cut and paste into align position in MS word so that it is appeared both sequences have similarity (Marchler-Bauer et al., 2017). For retrieving any nucleotide. we type the same at the NCBI port (www.ncbi.nlm.nih.gov/nucleotide or Protein) and to BLAST search to type the accession number for protein or DNA into BLAST port (https://blast.ncbi.nlm.nih.gov/Blast.cgi?PAGE_TYPE=BlastSearch) [Bairoch and Apweiler, 2000; Altschul, 1997]. 


\section{Result}

\section{Cobalamine biosynthesis genes in MDR-plasmids}

Vitamin- $\mathrm{B}_{12}$ is a very complex organic molecule and is needed for Haemoglobin protein to carry oxygen and to deliver it to cells and tissues. So we have checked if $c o b$ genes are present in MDR-plasmids of pathogens. Shewanella bicestrii plasmid pSHE-CTX-M (Accession no. CP022359, 193kb) contains a cobalamine biosynthesis protein (protein id. ASK71392) and an acetyl-COA carboxylase biotin carboxy carrier protein subunit (protein id. ASK71473; nt. 109480-110718C) including $m d r$ genes like CTX-M-15, aac3'-IIa, tetA, aph3"-Ib/d, and sull. Cobalamine biosynthetic protein has also detected in plasmids pKAZ2 and pKHM243 and similar DUF 3150 protein identified in many plasmids of E. coli, S. enterica, C. fruendii and uncultured bacterium (protein ids. WP_094198533, WP_065203424, BAS21640 and ALG87157). Plasmid pECAZ155_KPC of E. coli (accession no. CP019001; 272kb) has two cobalamine biosynthetic genes [potein ids. AQV87341 (261aa) and AQV87400 (425aa)] in associated with blaKPC, mphA, sull, aac6'II, aph6'-1d, blaCMY-2 and aac6'-1a mdr genes and drug efflux genes like MFS, tetB and cmlA (Chakraborty, 2016).

Plasmid K-109-R (154kb; accession no. KX029331) of Klebsiella pneumoniae had 12 mdr genes (bla, sul1/2, strAB, arr3, cat etc) with no vitamin synthesizing gene but when we analyzed the 25 unknown genes by BLAST, it appeared indeed it had cobalamine synthesizing gene (protein id. APD70537) with 99\% similarity to chromosomal sequences of many pathogens (protein ids. EEO11799, AKN19322, ALL42370) as well as plasmid (protein id. ARJ33497) and many DUF3150 domain proteins (protein id. CSO43544). Further, unknown protein (protein id. APD70542) appears a DNA methylase, unknown protein (id. APD70504) may be a DfrA family trimethoprim resistant protein (accession no. KX957972, protein id. APU91748) and unknown protein (protein id. APD70468) is related to NAD-dependent DNA ligase (protein id. SAA90237). Simple interpretation is that many unknown genes are vitamin metabolizing or related and might be assembled in many plasmids with no mdr genes but now quickly moved to MDR-plasmids to fulfil the symbiotic signals from host requiring, vitamins to support human metabolosome and conversely the nutrient for bacterial growth in the gastrointestinal biofilm. Similarly, after BLAST search of hundred of unknown proteins, we found cobT or vWF factor (protein ids. ASM79841 and APZ79706) in K. pneumoniae plasmids (accession nos. KY882285, 156kb and KX636095, 
$335 \mathrm{~kb}$ ) with a linked cobalamine DAF3150 domain biosynthesis protein. vWF factor (protein id. AKN19321) indentified in Salmonella enterica MDR plasmid pRH-1238 (accession no. KR091911, nt. 11696-109873) that also contained blaCMY-16, strA/B, floR, sul1/2, aphA6, aac6'-1b, mphA, qacE, blaNDM1 etc deadly $m d r$ genes (Villa L, Guerra B et al. 2015). FAD synthase (phospho hydrolase type, nt. 3669-2554) was located in p2964TF plasmid of $K$. pneumoniae (accession no. KT935446) and cobyric acid synthase (cobQ; protein id. ALU64791, nt. 19094-19879) and likely involved in riboflavin and cobalamine biosynthesis respectively (Bueno MF et al. 2016). NemN coproporphyrinogen III oxadase enzyme was acquired by Ensifer (protein id. AHK46764) and Rhizobium (protein id. PJI38304) species sharing $65 \%$ similarity.

\section{Thiamine Biosynthesis genes in MDR-plasmids}

Thiamine is vitamin B1 and thiamine pyrophosphate (TPP) is required in many biochemical reactions both for bacteria and human. So we checked the thi genes in large conjugative plasmids of pathogens. Large Enterobacter cloacae plasmid, p22ES-469 (Accession no. CM008897; 468kb) has thimine biosynthetic genes like thiH, thiA and thiC (protein ids. PIA01549, PIA01545, PIA01544 respectively) as well as enzymes involved in pantothenate metabolism (protein ids. PIA01523, PIA01562). However, no bla, sull, acc or aph mdr genes but drug transporters (protein ids. PIA01505, PIA01751, PIA01752). Plasmid pKOX_R1 contains thiF gene (protein id. AFN35065) for thiamine biosynthetic pathway including metal resistant genes and many MFS drug transporters as well as common $m d r$ genes like sull, aac3', ANT, aph and CTX-M-3 $\beta$-lactamase. Rhizobium leguminosarum plasmid (accession no. CP016287; 125kb) has thiamine biosynthetic genes (thiG/S/O/C; Protein ids. ANP89691/2/3/4) and cobW gene for cobalamine synthesis (protein id. ANP89796) but no $m d r$ gene but drug efflux genes. Large plasmids from same organism ( 1098kb; accession no. CP025013/ CP016287) have similar thiamine biosynthetic genes (protein id. ANP89582; AUW45994, AUW45995) and cobalamine biosynthetic genes (cobW; protein id. ANP89796). Such plasmids also have other vitamin biosynthetic genes like nicotinamide amidase (protein id. AUW45731; accession no. CP025013), biotin carboxylase and biotin sulfoxide reductase (776aa; protein id. AUW45720) suggesting absolute necessary signal indeed have generated in bacteria against high dose antibiotics and such plasmids have high numbers of $\mathrm{ABC}$ (protein id. AUW46396) and RND/MFS (protein id. AUW46415) 
transporters but no classical $m d r$ genes like tet, $\operatorname{str} A B$, amp, sul were found. It appears such plasmid is primitive and $m d r$ genes have generated lately and we have detected GNAT family $\mathrm{N}$-acetyl transferase (protein id. AUW46329) which is different from cat, aacC1 or aacA1 families acetylating enzymes. This data has suggested that accumulation of vitamin synthesizing enzymes into plasmids is not accidental and only is increasing in MDR-plasmid now due to complex antibiotic use.

\section{Folic acid biosynthetic genes in MDR-plasmids}

We also found folate and thimine metabolism enzymes in Sinorhizobium meliloti mega plasmid, pSymA (accession no. AE006469; 1354kb) like 5,10 methylene tetrahydrofolate reductase (protein id. AAK65825; nt. 1211022-1211975), formoyl tetrahydrofolate deformylase (protein id. AAK65824) and thiamine pyrophosphate binding enzyme (protein id. AAK65851) but no classical $m d r$ gene but penicillin binding proteins (protein ids. AAK65894, AAK65893) and many ABC or MFS transporters. This supports our hypothesis that bacteria will acquire more enzymes in the vitamin biosynthetic pathway to avert the action of antibiotics and will preserve the symbiotic relation in the intestine. So how and why such genes are now accumulating in MDR-plasmids is a important observation but needs research to elucidate if our hypothesis that all vitamin synthesizing enzymes will be in MDRplasmids to avert antibiotic actions on gut microbiota which are protected by symbiotic phenomenon during evolution (Wang et al., 2017).

\section{Biotin biosynthetic genes in MDR and Non-MDR plasmids}

An acetyl-COA carboxylase biotin carboxy carrier protein subunit (protein id. ASK71473; Accession no. CP022359, nt. 109480-110718C) located in Shewanella bicestrii plasmids. Rhizobium leguminosarum large plasmids (accession no. CP025013/ CP016287) have similar biotin carboxylase and biotin sulfoxide reductase (776aa; protein id. AUW45720) genes and also cobW genes involved in cobalamine biosynthesis. Ensifer adhaerens large plasmid pOV14b (1614kb) has 200 diversified ABC transporters and 60 diversified transcriptional regulators and few $m d r$ genes (blaOXA-like ; protein id. AHK47346), PBP1A (protein id. AHK47149) as well as acrB (protein id. AHK47037) and ermA/QacA (protein id. AHK47565) drug efflux proteins, Search indicated that the plasmid had biotin synthesizing enzymes (bioB, bioD and bioF) at the nt. 983383-981237 (protein ids. AHK47270, AHK4768 
and AHK47269 respectively). Thus multiple vitamin synthesizing genes are accumulating in mdr plasmids due to repeated use of complex antibiotics.

\section{Riboflavin biosynthetic genes in large MDR plasmids}

FAD synthase (phospho hydrolase type) was located in p2964TF plasmid of $K$. pneumoniae (accession no. KT935446; nt. 3669-2554). FAD-binding monooxygenase enzyme was reported in Ensifer plasmid pOV14b (protein id. AHK47466, nt. 1352625-1351060) as well as a FAD-dependent oxidoreductase (protein id. AHK47620).

\section{Pyrodoxine biosynthetic gene in MDR-plsmids}

A pyridoxine 5'-P oxidase (protein id. AHK47166) was reported in pOV14b large plasmid (accession no. CP007239) of Ensifer sp. and related to Sinorhizobium enzyme (65\% similarity, protein id. WP_058323781).

\section{Pantothenate biosynthetic gene in large MDR Plasmids}

Enterobacter cloacae plasmid, p22ES-469 (Accession no. CM008897) has acquired enzymes involved in pantothenate metabolism (protein ids. PIA01523, PIA01562) and also in association with thi genes and many $m d r$ genes.

\section{Niacin biosynthetic genes in MDR-plasmids}

Rhizobium leguminosarum large plasmids have nicotinamide amidase gene (protein id. AUW45731; accession no. CP025013, nt. 271544-272197) involved in niacin metabolism in association of thiG/O/C and cobW genes. $\mathrm{NH}_{3}$-dependent $\mathrm{NAD}^{+}$synthetase (nadE1, protein id. AHK46730) was located in Ensifer plasmid with $66 \%$ similarity to Agrobacterium enzyme (protein ids. CUX02012 and OFE24756) and a acyl coenzyme transferase (protein id. AHK47077) could be related to Rosebacter denitrificans (protein id. SFF70718, 77\% similarity). NADH-Ubiquinone oxido reductase (protein id. AHK47196) was found in Ensfer sp., large plasmid pOV14b (accession no. CP007239, nt. 854771-858262).

\section{Many biosynthetic genes in large MDR plasmids}

As discussed earlier, we detected many large plasmids have multiple vit genes. Enterobacter cloacae large plasmid p22ES-469 has many thi and pan genes (Miranda-Ríos et al., 1997). Shewanella bicestrii plasmid pSHE-CTX-M has cob and rib genes. Rhizobium leguminosarum very large plasmid also have multiple thi genes as well as bio and cob genes 
(accession no. CP025013). This implies that gradual selection of vit genes into plasmids have initiated and maintained during antibiotic exposure.

\section{Discussion}

Multi-resistance horrors have intensified worldwide. Indian NAP-AMR (The National Action Plan on Antimicrobial Resistance- 2017-2021) has pinpointed five main areas: (i) improving awareness and understanding of AMR through effective education and surveillance, (ii) reducing infection by increasing preventive measures, (iv) reducing the use of antimicrobials in health, food animals and agriculture (v) promoting for AMR research and drug innovations and (v) strengthening India's leadership on AMR and International collaboration (assessed on October, 2017; Chandy et al., 2014). We thus have studied superbugs at the molecular level. Sadly, study indicated that huge antibiotics use did influenced $2 \times 10^{12}$ bacteria in the intestine at the molecular level altering vitamin synthesizing capacity and critical $m d r$ genes including vitamin synthesizing genes are created in plasmids to save bacteria and human under symbiosis. Indeed high oral dose of ampicillin, streptomycin , ciprofloxacin and tetracycline had killed all gut bacteria between 1950-1970 before the introduction of B-complex vitamin capsule and bifidobacterrium probiotic capsule (Feng et al., 2016). Thus it appears multidrug-resistant genes creation is to protect microbiota from repeated doses of antibiotics that we have consumed since 1940s. We detected blaTEM-1, blaCTX-M-15, blaNDM-1 and aac6'-1b mdr genes in Ganga River water and Digha sea water and such superbugs are resistant to many drugs like imipenem, meropenem, amikacin and linezolid. We used advanced molecular tools like PCR and DNA sequencing to prove the fact. Further, several high quality research from US Human Microbiome Project (HMP), European Metagenomics of the Human Intestinal Tract (MetaHIT) and others have demonstrated the beneficial functions of the normal gut flora (>35000 species) on health. Symbiotic bacteria contribute to the functional biodiversity in the aquatic world and influence the fitness of the host organisms and microhabitats ecosystem. It has been suggested that LPS, vitamins and butyrate from intestinal bacteria activate luminal cells to secrete interleukins and cytokines that help to synthesis diversified $m d r$ genes in conjugative plasmids and chromosome to protect gut microbiota against action of high dose of antibiotics (Gibson et al., 2016). We propose that MDR bacteria will be normal resident of intestine until we stop oral antibiotic use. It is thus G-20 Nations in Germany are united for active research on MDR bacteria to stop superbug horror and WHO has warned to use multiple doses of antibiotics to patients and animals. Many microorganisms are also gathering toxin genes in 
plasmids and likely very threatening to public. Bacillus thuringiensis plasmid pBMB293 (Accession no. CP007615, 294kb) has no $m d r$ gene but genes for enterotoxins (protein id. AIM34697), dipterans toxin (protein id. AIM34741) and reverse transcriptase, DNA polymerase $\beta$, DNA topoisomerase III and type II secretion system. Similarly, Bacillus anthrus plasmid pX01 (accession no. CM002399; 171kb) has toxin gene (protein id. AFL55645, 809aa) and also in pBMB293 plasmid. We need to reduce global toxicity in water and our industries must know the knowledge of contamination of chemicals and heavy metals in water increasing superbugs spread.

\section{Conclusion}

WHO has suggested to follow AMR Action Plan and G-20 leaders and Scientists have agreed to reduce antibiotics use in human, animal, and agricultural land as well as to augment research on novel therapeutics alternate to antibiotics (Villa et al., 2015). We have purified organic phyto-extracts (Cassia fistula, Suregada multiflora, Syzygium aromaticum, Cinnamomum zeynalicum, etc) that inhibit Kolkata superbugs and gives a hope for new drug development (Chakraborty, 2015). We slogan, "Come Back to Nature: Save Plants and Use as Medicine". Many technologies like enzybiotics, phage therapy and gene medicines are under development other than herbal therapy (Kutter et al., 2015). Sadly, household water, sea water, rain water and river water are contaminated greatly with superbugs increasing deadly infections where MRSA Staphylococcus aureus, MDR Acinetobacter baumannii and NDM1 Escherichia coli infections are all antibiotic resistant. Thus we lost our win position using ampicillin, streptomycin, tetracycline, ciprofloxacin, azithromycin, and cefotaxime and imipenem drugs against MDR bacterial infections. The GenBank data analysis has proved our hypothesis that vitamin crisis in human due to antibiotic therapy. We think much analysis of unknown genes in superbug plasmids are necessary and likely NIH (NCBI, USA) should act quickly. Perhaps DNA recombination enzymes like tranposases, resolvases, integrases, topoisomerases and plasmids partition enzymes are good target for development of therapeutics against superbugs. Trully, all famous drug companies are in horror to invest in new antibiotic discovery. But phage therapy (bacteriophages $\mathrm{T} 4 / \theta / \phi$ ), enzybiotics (lysin, lysozyme), gene medicines (ribozyme, CASPER-CAS, IL gene therapy) and delivery of toxic drugs efficiently using nano-carriers (fullerenes, DNA-Origami) are frontier medical sciences to regulate the $21^{\text {st }}$ Century drug industry (Dunbar et al., 2018). This work may also through 
some highlight into combat measure and novel drug design against superbugs and there is no similar report in the Pubmed database (Xu et al., 2014).

\section{Acknowledgement}

We thank Profs. Amiya Panda and Chandradipa Ghosh of Vidyasagar University; Dr. Sujoy Dasgupta of Bose Institute, Kolkata; Dr. Ananta Ghosh of IIT-Kharagpur and Dr. Ramdhan Mazi of Indian Institute of Chemical Biology, Kolkata for help during the study.

\section{References}

Abraham EP, Chain E (1988). An enzyme from bacteria able to destroy penicillin. 1940. Rev Infect Dis., 10: 677-678.

Ahmadjian PS. (2000). Symbiosis: An introduction to biological associations. $2^{\text {nd }}$ edition. Oxford University Press. (ISBN 0-19-511807-3).

Ausubel FM, Brent R., et al., (1989). In Current Protocols in Molecular Biology. Greene publishing associates and Wiley-Interscience, New York.

Bairoch A, Apweiler R (2000). The SWISS-PROT protein sequence database and its supplement TrEMBL in 2000. Nucleic Acids Res., 28:45-48.

Bush K, Jacoby, G. A., (2010). Updated functional classification of $\beta$-lactamases. Antimicrob. Agents Chemother., 54: 969-976.

Carattoli A., (2009). Resistance plasmid families in Enterobacteriaceae. Antimicrob Agents Chemother, 53: 2227-2238.

Chakraborty AK., (2015). High mode contamination of multi-drug resistant bacteria in Kolkata: mechanism of gene activation and remedy by heterogenous phyto-antibiotics. Indian J Biotechnol. 14, 149-159.

Chakraborty AK., (2017a) Mechanisms of AMR: Bacteria won the battle against antibiotics. Insights Biomed. 2 (4), 19. Doi.10.21767/2572-5610.100034.

Chakraborty AK., (2017b). Multi-drug resistant bacteria from Kolkata Ganga river with heterogeneous MDR genes have four hallmarks of cancer cells but could be controlled by organic phyto-extracts. Biochem. Biotechnol. Res., 5(1): 11-23. 
Chakraborty AK., (2017c). Colistin drug resistant determinant Mcr-1 gene spreads in conjugative plasmids creating huge confusion for the treatment of multi-drug resistant infections. Ame. Res. J. Biotechnol., 1(1): 1-9.

Chakraborty AK., (2017d). Mechanism of AMR: Mdr genes and antibiotics decoys retard the new antibiotic discovery against superbugs. Nov Appro Drug Des Dev. 2(1): 555576.

Chakraborty AK., (2017e). Enzybiotics, a new class of antimicrobials targeted against multidrug-resistant superbugs. Nov Appo Drug Des Dev. 2(4): 555592.

Chakraborty AK., (2017f) MDR genes are created and transmitted in plasmids and chromosomes to keep normal intestinal microbiota alive against high dose antibiotics- a hypothesis. J Mol Med Clin Appl 2(1): 109. Doi: http://dx.doi. org/10.16966/2575-0305.109.

Chakraborty AK, Maity M, Patra S, Mukherjee S, Mandal T (2017). Complexity, heterogeneity and mutational analysis of antibiotic inactivating acetyl transferases in MDR conjugative plasmids conferring multi-resistance. Res Rev: J Microbiol Biotechnol., 6(2): 2843.

Chakraborty AK, Roy T, Mondal S., (2016). Development of DNA nanotechnology and uses in molecular medicine and biology. Insights Biomed., 1(2): 13.

Chakraborty AK., (2016a). Multi-drug resistant genes in bacteria and $21^{\text {st }}$ Century problems associated with antibiotic therapy. Biotechnol Ind J. 12(7): 114.

Chakraborty AK., (2016b). Complexity, heterogeneity, 3-D structures and transcriptional activation of multi-drug resistant clinically relevant bacterial beta-lactamases. Trends Biotechnol-open access. 2 (1): 1-001.

Chakraborty AK., (2016c). In silico analysis of hotspot mutations in the bacterial NDM-1 and KPC-1 carbapenemases that cause severe MDR phenotypes. Biochem Biotechnol Res. 4(1): 17-26.

Chakraborty AK, Zink MA, Boman BM, Hodgson CP., (1993). Synthetic Retrotransposon vectors for Gene Therapy. FASEB J., 7: 971-977.

Chakraborty AK, Cichutek K, Duesberg PH., (1991). Transforming function of proto-ras genes depends on heterologous promoters and is enhanced by specific point mutations. Proc. Natl. Acad. Sci. USA. 88: 2217-2221.

Chakraborty AK, Hodgson CP., (1998). Role of far upstream repressor elements controlling the protoHa-ras gene transcription. Biochem. Biophys. Res. Commun. 252: 716-722.

Chandy SJ, Michael JS, Veeraraghavan B et al., (2014). ICMR Programme on Antibiotic Stewarship, Prevention of Infection \& Control (ASPIC). Ind J Med Res. 139: 226-230.

Dallas WS et al., (1992). Cloning, Sequencing, and Enhanced Expression of the Dihydropteroate Synthase Gene of Escherichia coli MC4100. J Bacteriol., 174(18): 59615970. 
Dunbar CE, High $\quad$ KA, Joung JK, Kohn $\quad$ DB, Ozawa K, Sadelain $\quad$ M. (2018). Gene therapy comes of age. Science. 359(6372). pii: eaan4672. doi: 10.1126/science.aan4672.

Feng Y, Yang P, Wang X, Zong Z. (2016). Characterization of Acinetobacter johnsonii isolate XBB1 carrying nine plasmids and encoding NDM-1, OXA-58 and PER-1 by genome sequencing. J Antimicrob Chemother., 71: 71-75. doi: 10.1093/jac/dkv324.

Flach CF., Johnning,A., Nilsson,I., Smalla,K., Kristiansson,E, Larsson,D.G. (2015). Isolation of novel IncA/C and IncN fluoroquinolone resistance plasmids from an antibiotic-polluted lake. J Antimicrob Chemother. 70 (10): 2709-2717.

Fosberg KJ, Reyes A, Wang B., et al. (2012). The shared antibiotic resistome of soil bacteria and human pathogens. Science, 337:1107-1111.

Gibson MK, Wang B, Ahmadi S, Burnham GA,. Tarr PI, Warne BB, Dantas G. (2016). Developmental dynamics of the preterm infant gut microbiota and antibiotic resistome. Nat Microbiol., 1: 16024. doi:10.1038/nmicrobiol.2016.24.

Grossart H-P, Riemann L, Tang KW. (2013). Molecular and functional ecology of aquatic microbial symbionts. Front Microbiol., 4: 59. Doi: 10.3389/fmicb.2013.00059.

Gunguly NK, Arora NK, Chandy SJ et al., (2011). Antibiotic Resistance Partnership (GARPIndia Working Group. Rationalising antibiotic use to limit antibiotic resistance in Inda. Ind J Med Res., 134: 281-294.

Harme CJ., Holt,K.E. and Hall,R.M. (2015). A type 2 A/C2 plasmid carrying the aacC4 apramycin resistance gene and the erm(42) erythromycin resistance gene recovered from two Salmonella enterica serovars. J Antimicrob Chemother., 70 (4): 1021-1025.

Hazra AB, Han AW, MehtaAP et al., (2015). Anaerobic biosynthesis of the lower ligand of vitamin B12. Proc Natl Acad Sci USA. 112 (34): 10792-10797.

Huang TW, Wang JT, Lauderdale TL et al., (2013). Complete sequences of two plasmids in a blaNDM-1-positive Klebsiella oxytoca isolate from Taiwan. Antimicrob. Agents Chemother. 57 (8): 4072-4076.

Ho PL, Lo WU, Yeung MK et al., (2011). Complete sequencing of pNDM-HK encoding NDM-1 carbapenemase from a multidrug-resistant Escherichia coli strain isolated in Hong Kong. PLoS One., 6 (3): E17989.

Ibiene AA et al., (2011). Multidrug-resistant (MDR) bacteria isolated from different drinking water sources. New York Science J., 4 (12): 50-56.

Johnson M, Zaretskaya I, Raytselis Y, Merezhuk Y, McGinnis S, Madden TL. (2008). NCBI BLAST: a better web interface. Nucleic Acids Res, 36 (suppl 2):W5-W9.

Karmakar S, Biswas D, Shaikan NM, Chatterjee SK, Kataria VK, Kumar R. (1991). Role of a large plasmid of Salmonella typhi encoding multiple drug resistance. J Med Microbiol., 34: 149-151. 
Kroft BS et al., (2013). Draft Genome Sequences of Two Salmonella strains from the SARA Collection, SARA64 (Muenchen) and SARA33 (Heidelberg), provide insight into their antibiotic resistance. Genome Announc 1 (5): e00806-13.

Kutter EM, Kuhl SJ, Abedon ST. (2015). Re-establishing a place for phage therapy in western medicine. Future Microbiol., 10: 685-688.

Laxminarayan R, Duse A, Wattal C et al., (2013). Antibiotic Resistance- the need for Global Solutions. Lancet Infect Dis., 13: 1057-1098.

Le Chatelier, E., et al. (2013). Richness of human gut microbiome correlates with metabolic markers. Nature, 500, 541-546.

Lopez P, Espinosa M, Greenberg B and Lacks S. (1987). Sulfonamide resistance in Streptococcus pneumoniae: DNA sequence of the gene encoding dihydropteroate synthase and characterization of the enzyme. J Bacteriol., 169: 4320-4326.

Marchler-Bauer A et al. (2017), "CDD/SPARCLE: functional classification of proteins via subfamily domain architectures.", Nucleic Acids Res.45(D): 200-203

Mataseje LF, Peirano G, Church DL, Conly J, Mulvey M, Pitout JD (2016). Colistin nonsusceptible Pseudomonas aeruginosa ST654 with blaNDM-1 arrives in North America. Antimicrob Agents Chemother. Jan 11. pii: AAC.02591-15.

Mckenna M (2013) The last resort: Health officials are watching in horror as bacteria become resistant to powerful carbapenem antibiotics-one of the last drug on the self. Nature 499: 394396.

McMurry L, Petrucci R, Levy S.(1980). Active efflux of tetracycline encoded by four genetically different tetracycline resistance determinants in Escherichia coli. Proc. Natl.

Acad. Sci. U.S.A, 77: 3974-3977.

Merlo TP, Dabul AN, Camargo IL. (2015). Different VanA elements in E. faecalis and in E. faecium suggest at least two origins of Tn1546 among VRE in a Brazilian Hospital. Microb Drug Resist., 21(3): 320-328.

Miranda-Ríos J, Morera C, Taboada H, Dávalos A, Encarnación S, Mora J, Soberón M. (1997). Expression of thiamin biosynthetic genes (thiCOGE) and production of symbiotic terminal oxidase cbb3 in Rhizobium etli. J Bacteriol. 179(22): 6887-6893.

Morten O, Sommer A, Dantas G, and Church GM. (2009). Functional characterization of the antibiotic resistance reservoir in the human microflora. Science, 325(5944): 1128-1131.

McArthur AG, Waglechner N, Nizam F et al., (2013). The Comprehensive Antibiotic Resistance Database. Antimicrob Agents Chemther., 57 (7): 3348-3357. 
Paul D, Dhar D, Maurya AP et al., (2015). Co-carriage of blaKPC-2 and blaNDM-1 in Clinical Isolates of Pseudomonas aeruginosa associated with Hospital infections from India. PLoS One 10(12): e0145823. doi:10.1371/journal.pone.0145823.

Pathak SP et al., (1993) Seasonal variation in survival and antibiotic resistance among various bacterial populations in a tropical river. J Gen Appl Microbial., 39 (1): 47-46.

Reynolds PE (1989) Structure, biochemistry and mechanism of action of glycopeptides antibiotics. Eur J Clini Microbiol Infect Dis.. 8: 943-950.

Robicsek A, Strahilevitz J, Jacoby G. A et al., (2006). Fluoroquinolone modifying enzyme: a new adaptation of a common aminoglycoside acetyltransferase. Nat Med., 12: 83-88.

Rodionov DA, Vitreschak AG, Mironov AA, Gelfand MS. (2002). Comparative genomics of thiamin biosynthesis in procaryotes. New genes and regulatory mechanisms. J Biol Chem. 277(50): 48949-48959.

Sambrook J, Fritsch EF, Maniatis T. (1989). Molecular Cloning: A Laboratory Manual. $2^{\text {nd }}$ ed. Cold Spring Harbor Laboratory Press, New York.

Sanger F, Nicklen S, Coulson AR. (1977). DNA sequencing with chain-terminating inhibitors. Proc Natl Acad Sci USA., 74: 5463-5467.

Shaw KJ, Rather PN, Hare RS and Miller GH. (1993). Molecular genetics of aminoglycoside resistance genes and familial relationships of the aminoglycoside-modifying enzymes. Microbiol Reviews, 57(1): 138-163.

Schwarz S, Kehrenberg C, Doublet, B and Cloeckaert A. (2004). Molecular basis of bacterial resistance to chloramphenicol and florfenicol, FEMS Microbiol Rev., 28: 519-542.

Stiffler MA, Hekstra DR and Ranganathan R., (2015). Evolvability as a function of purifying selection in TEM-1 $\beta$-lactamase. Cell, 160: 882-892.

Sun J, Deng Z, Yan A (2014). Bacterial multidrug efflux pumps: Mechanisms, physiology and pharmacological exploitation. Biochem Biophys Res Communi., 453: 254-267.

Talarico TL, Dev IK, Dallas WS, Ferone R, Ray PH. (1991). Purification and partial characterization of 7,8-dihydro-6-hydroxymethylpterin-pyrophosphokinase and 7,8dihydropteroate synthase from Escherichia coli MC4100. J Bacteriol., 173: 7029-7032.

Vander Horn PB, Backstrom AD, Stewart V, Begley TP. (1993). Structural genes for thiamine biosynthetic enzymes (thiCEFGH) in Escherichia coli K-12. J Bacteriol., 175(4): 982-992.

Villa L, Guerra B, Schmoger S et al., (2015). New plasmid-mediated aminoglycoside 6'-Nacetyltransferase, AAC(6')-Ian, and ESBL, TLA-3, from a Serratia marcescens clinical isolate. J. Antimicrob. Chemother. 70 (5): 1331-1337 
Wang X., Lu M., Shi Y., Qu Y, Cheng X., (2015). Discovery of novel new Delhi metallo- $\beta$ lactamases inhibitors by multistep virtual screening. PLoS One, 10: e0118290.

Wang L, Fang H, Feng J et al. (2015). Complete sequences of KPC-2 encoding p628-KPC and CTX-M-65 encoding p628-CTXM coexisted in Klebsiella kneumoniae. Frontier Microbiol., 6: 838.

Wang J, Li Y, Xu X., et al., (2017). Antimicrobial resistance of Salmonella enterica Serovar Typhimurium in Shanghai, China. Front Microbiol., 8: 510. doi: 10.3389/fmicb. 2017.00510.

Xu ZQ, Flavin MT, Flavin J., (2014). Combating multi-drug resistant gram-negative bacterial infection. Exp Opini Investi Drugs, 23: 163-182.

Figures 

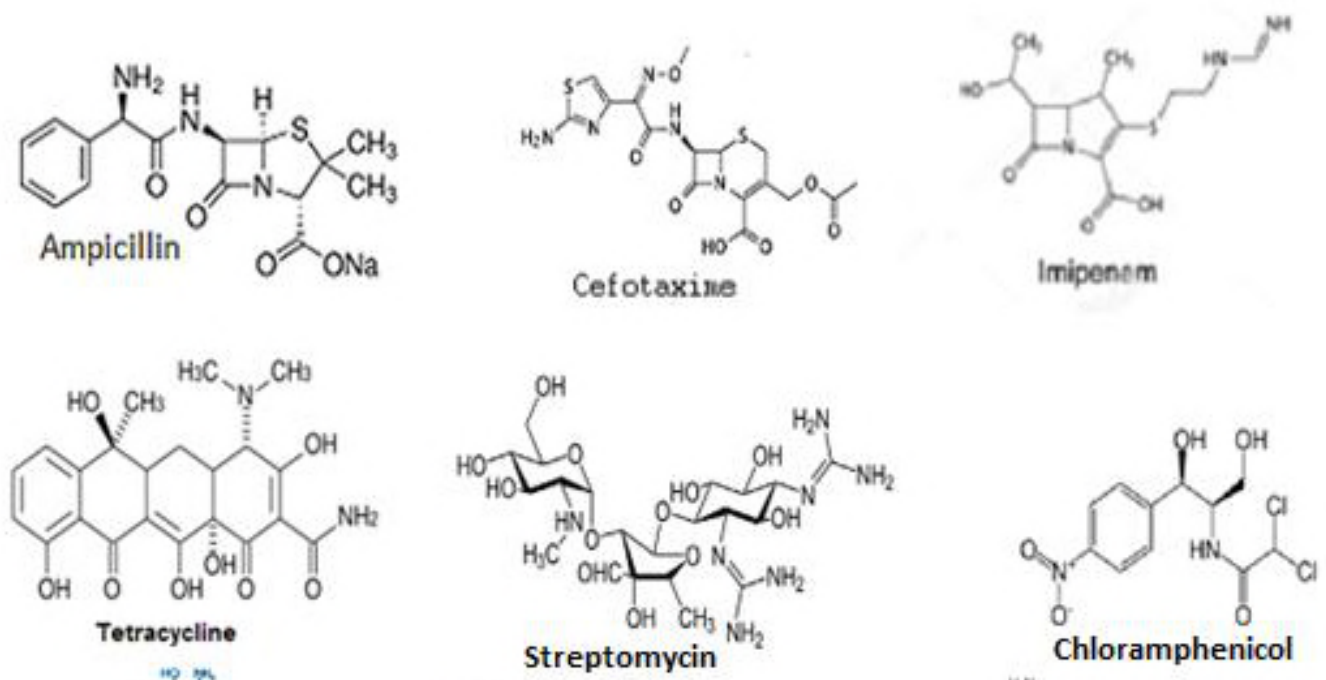

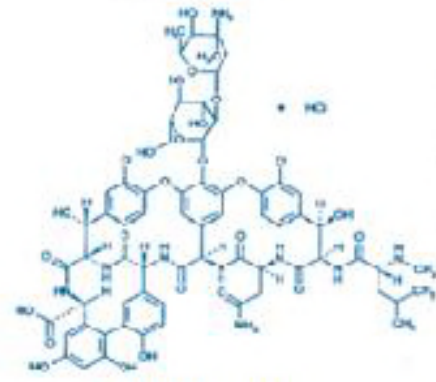

Vancomycin

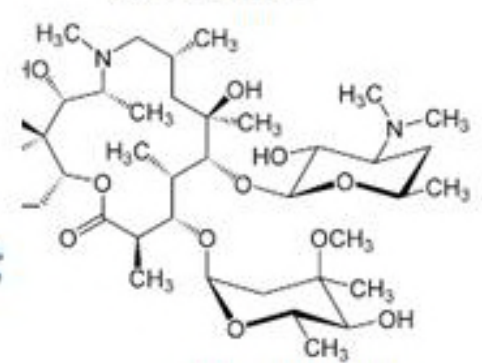

Azithromycin

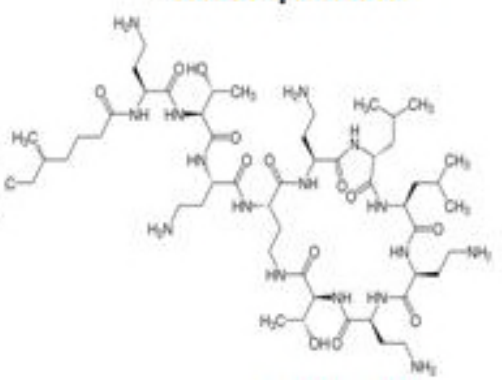

Colistin

Fig. 1. Complex structures of antibiotics that are destroyed by mdr genes and now useless in superbug infections. 


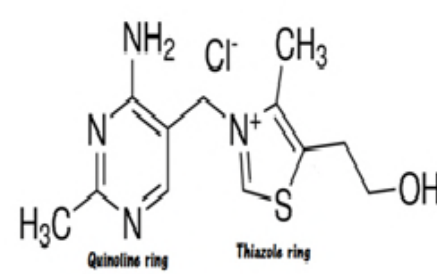

Thamine (BI)
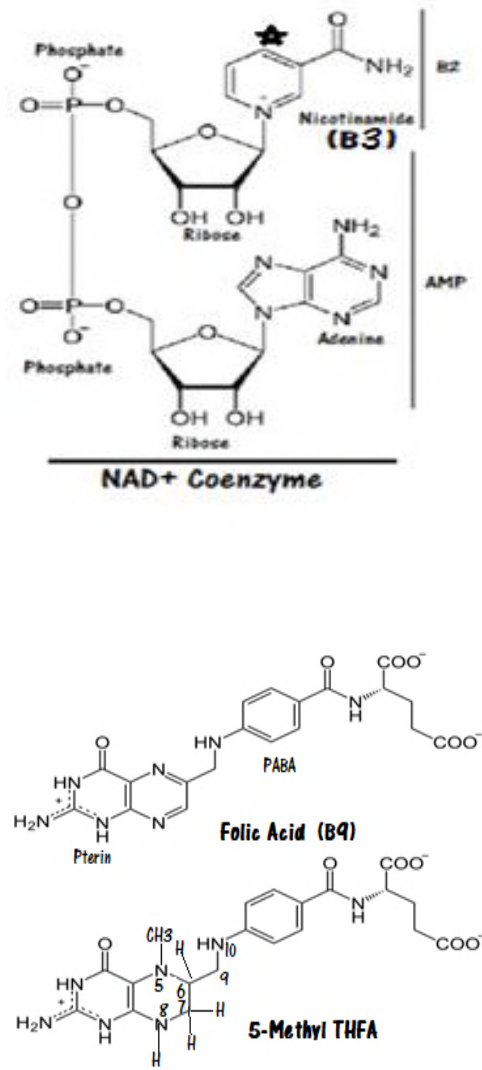
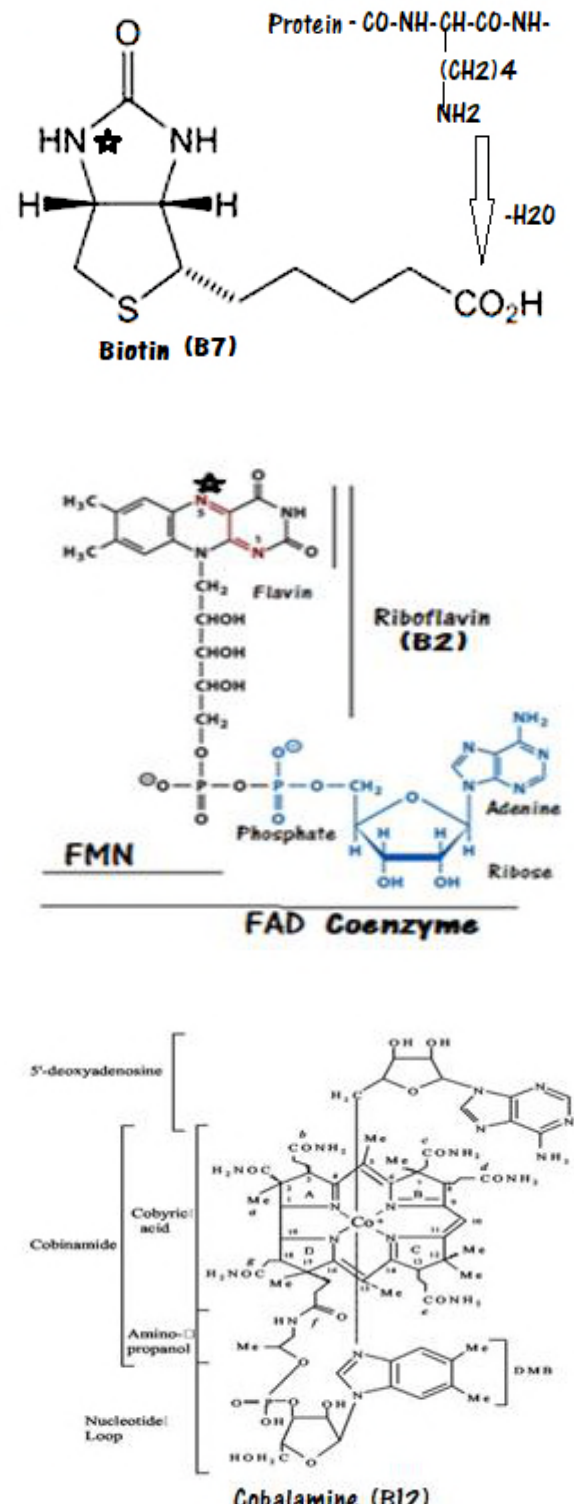

Cobalamine (B12)

Fig.2. Complex structure of vitamins that intestinal bacteria do synthesis for human. 


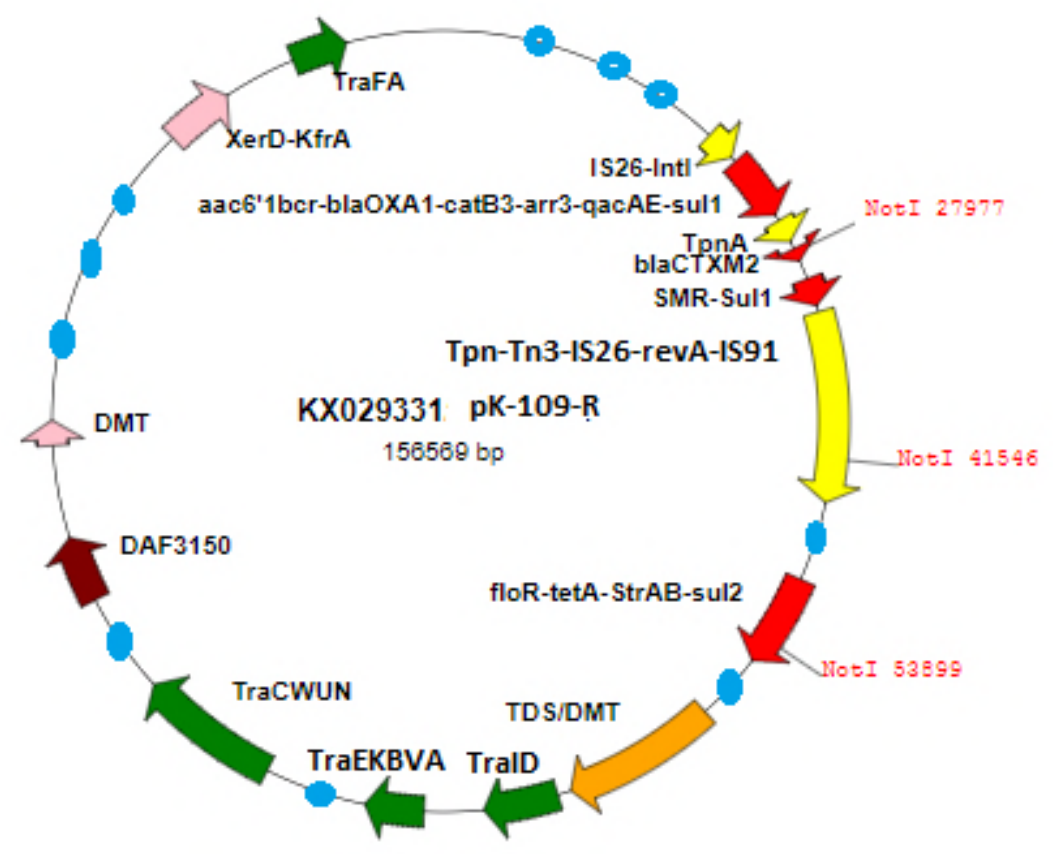

Fig.3. Structure of recent MDR-plasmid pK-109-R (accession no. KX029331). Green is Tra genes involved in conjugation; Red is $m d r$ genes, Yellow is transposons, Blue is unknown genes and IS elements, and maroon is vitamin synthesizing genes (DAF3150). Restriction enzyme Not1 (octa-cutters) sites are also seen. Such plasmids are capable of donating mdr genes to orther bacteria came to close contact and thus most bacteria became drug resistant. 


\begin{tabular}{|c|c|c|c|c|c|}
\hline Vit genes & PLASMID & Bacteria (Genus) & $\begin{array}{l}\text { Accession } \\
\text { no }\end{array}$ & Protein id & Enzyme name and function \\
\hline thiA & p22ES-469, 469kb & Enterobacter & CM008897 & PIA01545, 213aa & Thiamine phosphate synthase \\
\hline thiH & p22ES-469, 469kb & Enterobacter & CM008897 & PIA01549, 375aa & 2-imino acetate synthase \\
\hline thiI & pSymA & Sinorhizobium & AE006469 & AAK65851, 566аa & Thiamine-PP binding protein \\
\hline thiG & $\begin{array}{l}\text { P1, 125kb } \\
\text { pRLN1, 110kb }\end{array}$ & $\begin{array}{l}\text { Rizobium } \\
\text { Rizobium }\end{array}$ & $\begin{array}{l}\text { CP016287 } \\
\text { CP025013 }\end{array}$ & $\begin{array}{l}\text { ANP89691, 257aa } \\
\text { AUW45996, 257aa }\end{array}$ & $\begin{array}{l}\text { Catalyses the formation of } \\
\text { thiazole. EC: } 1.5 .3 .8 \text {. }\end{array}$ \\
\hline thiS & $\mathrm{P} 1,125 \mathrm{~kb}$ & Rizobium & CP016287 & ANP89692, 65aa & $\begin{array}{l}\text { Ubiquitin-like sulphur carrier } \\
\text { protein }\end{array}$ \\
\hline thiO & $\mathrm{P} 1,125 \mathrm{~kb}$ & Rizobium & CP016287 & ANP89693, 329aа & FAD-dependentoxido-reductase \\
\hline thiC & $\begin{array}{l}\text { P1, 125kb } \\
\text { pRLN1, 110kb } \\
\text { p22ES-469, 469kb }\end{array}$ & $\begin{array}{l}\text { Rizobium } \\
\text { Rizobium } \\
\text { Enterobacter }\end{array}$ & $\begin{array}{l}\text { CP016287 } \\
\text { CP025013 } \\
\text { CM008897 }\end{array}$ & $\begin{array}{l}\text { ANP89694, 607aa } \\
\text { AUW45993, 607aa } \\
\text { PIA01544, 631aa }\end{array}$ & $\begin{array}{l}\text { Phosphomethyl pyrimidine } \\
\text { synthase }\end{array}$ \\
\hline thiF & $\begin{array}{l}\text { pKOX_R1, 354kb } \\
\text { pC13-2, 104kb }\end{array}$ & $\begin{array}{l}\text { Klebsiella } \\
\text { Acinetobacter }\end{array}$ & $\begin{array}{l}\text { CP003684 } \\
\text { KU549175 }\end{array}$ & $\begin{array}{l}\text { AFN35065, 742aa } \\
\text { AMQ95348, 576aа }\end{array}$ & Thiamine synthetase \\
\hline thiE & $\begin{array}{l}\text { P2, 181kb } \\
\text { pRLN1, 110kb }\end{array}$ & $\begin{array}{l}\text { Vibrio } \\
\text { Rizobium }\end{array}$ & $\begin{array}{l}\mathrm{CP} 022555 \\
\mathrm{CP} 025013\end{array}$ & $\begin{array}{l}\text { ASQ38758, 204aa } \\
\text { AUW45997, 202aa }\end{array}$ & $\begin{array}{l}\text { Thiamine phosphate } \\
\text { pyrophosphorylase }\end{array}$ \\
\hline $\operatorname{cobW}$ & $\mathrm{P} 1,125 \mathrm{~kb}$ & Rizobium & CP016287 & ANP89796 & Cobalaminebiosynthetic protein \\
\hline $\operatorname{cobX}$ & $\begin{array}{l}\text { pKHM-1, 166kb } \\
\text { pVPS129, 157kb } \\
\text { pRJ119-NDM1, 335kb } \\
\text { pECAZ155_KPC, 272kb }\end{array}$ & $\begin{array}{l}\text { Citrobacter } \\
\text { Vibrio } \\
\text { Klebsiella } \\
\text { Escherichia }\end{array}$ & $\begin{array}{l}\text { AP014939 } \\
\text { KY014464 } \\
\text { KX636095 } \\
\text { CP019001 }\end{array}$ & $\begin{array}{l}\text { BAS21640, 425aa } \\
\text { ARJ33497, 425aa } \\
\text { APZ79705, 425aa } \\
\text { AQV87400, 425aa }\end{array}$ & $\begin{array}{l}\text { DUF3150 domain protein. } \\
\text { Na_Ca_ex domain synthesizing } \\
\text { genes }\end{array}$ \\
\hline $\operatorname{cob} Q$ & pECAZ155_KPC, 272kb & Escherichia & CP019001 & AQV87341, 261aa & Cobalaminebiosynthetic protein \\
\hline $\operatorname{cobS}$ & pRJ119-NDM1, 335kb & Klebsiella & KX636095 & APZ79701, 332aa & Cobalamine chelatase \\
\hline thfR & pSymA, $135 \mathrm{~kb}$ & Sinorhizobium & AE006469 & AAK65825, 317aa & $\begin{array}{l}5,10 \text { methylene tetrahydrofolate } \\
\text { reductase, EC: } 1.5 .1 .20\end{array}$ \\
\hline thfD & pSymA, $135 \mathrm{~kb}$ & Sinorhizobium & AE006469 & AAK65824, 286aа & $\begin{array}{l}\text { Formoyl tetrahydrofolate } \\
\text { deformylase, EC:3.5.1.10 }\end{array}$ \\
\hline$p d x H$ & $\mathrm{P} 2,181 \mathrm{~kb}$ & Vibrio & CP022555 & ASQ38911, 211aa & $\begin{array}{l}\text { pyridoxamine } 5 \text { '-phosphate } \\
\text { oxidase }\end{array}$ \\
\hline bioc & $\mathrm{pCB} 782,156 \mathrm{~kb}$ & Rizobium & CP007068 & AHG48429,464aa & biotin carboxylase \\
\hline bioD & pRLN1, 110kb & Rizobium & CP025013 & AUW45720, 776aa & biotin sulfoxide reductase \\
\hline biot & pSHE-CTX-M, 193kb & Shewanella & CP022359 & ASK71473, 412aa & $\begin{array}{l}\text { acetyl-COA carboxylase biotin } \\
\text { carboxy carrier protein }\end{array}$ \\
\hline nicA & pRLN1, 110kb & Rizobium & CP025013 & AUW45731, 217aa & Nicotinamide amidase \\
\hline
\end{tabular}

Table-1. Identification of Vitamin Synthesizing Genes in MDR-plasmids with drug efflux and mdr genes. Rizobium plasmids have more drug efflux and ABC transporters but less MDR genes. 